Research Article

\title{
Application of Spherical Magnetic Bearing in Magnetically Suspended Control and Sensitive Gyro
}

\author{
Yin Zengyuan $\left(\mathbb{D},{ }^{1}\right.$ Yuanwen Cai, ${ }^{1}$ Bing Liu, ${ }^{2}$ Wang Weijie, ${ }^{1}$ and Xiaocen Chen ${ }^{1,2}$ \\ ${ }^{1}$ Space Engineering University, Beijing 101416, China \\ ${ }^{2}$ Beijing Institute of Special Electromechanical Technology, Beijing, 100012, China \\ Correspondence should be addressed to Yin Zengyuan; freeyzy1@163.com
}

Received 8 October 2019; Accepted 9 March 2020; Published 15 May 2020

Academic Editor: Nunzio Salerno

Copyright (c) 2020 Yin Zengyuan et al. This is an open access article distributed under the Creative Commons Attribution License, which permits unrestricted use, distribution, and reproduction in any medium, provided the original work is properly cited.

\begin{abstract}
This paper presents a spherical magnetic bearing and analyzes its application in magnetically suspended control and sensitive gyro (MSCSG). The main advantage of the spherical magnetic bearing is that it can eliminate the interference torque in the process of rotor tilt and improve the suspension accuracy of the rotor. By comparing the finite element analysis results of spherical magnetic bearing and traditional cylindrical magnetic bearing, the interference torque to the rotor of cylindrical magnetic bearing increases gradually with the increase of the rotor tilt angle. The interference torque to the rotor of spherical magnetic bearing does not change with the rotor tilt, and the interference torque is basically $0 \mathrm{Nm}$. Then, dynamic and static experiments were carried out to verify the interference torque generated in the process of spherical magnetic suspended rotor tilting. The experimental results show that the runout value of the rotor's translation and tilt signals in both static and dynamic states is basically consistent with the value of the equilibrium state of the rotor (tilt angle $=0$ ). Therefore, spherical magnetic bearing will not produce interference torque, which is of great significance for realizing the high-precision control of magnetic suspension inertia mechanism.
\end{abstract}

\section{Introduction}

Magnetic bearing has the advantages of active vibration suppression, no friction, no lubrication, high speed, long life, and so on [1-4]. The magnetic suspension inertia actuator supported by magnetic bearing can eliminate the contact friction and wear of mechanical bearing and has the advantages of high torque accuracy, microvibration, and active vibration suppression [5-7]. Therefore, magnetic bearing technology is widely used in magnetic suspension inertia actuator [8].

The main performance of magnetic bearing is to keep the rotor suspended in the air stably [9-11]. The performance of magnetic bearing mainly depends on the suspension effect of the rotor. Despite the mature development of magnetic bearings, such as active magnetic bearings (AMBs), passive magnetic bearings (PMBs), and permanent-magnet-biased hybrid magnetic bearings (HMBs), each of which has advantages and disadvantages and has been well used, the structure of magnetic bearings is basically cylindrical magnetic pole structure $[12,13]$. Cylindrical magnetic bearing is convenient for processing and assembly. The disadvantage of using cylindrical magnetic bearing is that (1) the magnetic suspension rotor cannot achieve large angle tilt, which limits the output of control torque; (2) the rotor will generate interference torque during the tilt process, which seriously affects the accuracy of MSCSG output torque.

Traditional magnetic bearings are cylindrical structures. For example, the magnetic bearings in reference [14-17] all adopt cylindrical structure. The main advantage of the spherical magnetic bearing is that it can eliminate the interference torque in the process of rotor tilt and improve the suspension accuracy of the rotor. However, for cylindrical magnetic bearings, the force on the rotor points to the normal direction of the rotor, which leads to the existence of force arms between the electromagnetic forces, thus causing the rotor to bear torque. The torque generated by the stator causes the rotor to tilt, which reduces the control accuracy of 
the rotor and affects the performance of the magnetic suspension inertia mechanism.

In reference [17], a novel conical active magnetic bearing with claw structure is proposed. The characteristic of this structure is that a single magnetic bearing can realize the 3DOF motion of the rotor. In addition, the radial magnetic bearing is installed in the axial direction, which saves the radial dimension of the mechanism. However, the force generated by the stator to the rotor is still perpendicular to the normal direction of the conical surface of the rotor. The rotor of this structure has a smaller radial size. Although the smaller radial size can reduce the force arm of the electromagnetic force, when the rotor tilts, the rotor is still subject to interference torque, which will result in poor suspension accuracy. Wang [18] proposed a 3-DOF conical magnetic bearing. The advantage of this structure is that the three degrees of freedom translation of the rotor is decoupled. However, the electromagnetic force of the rotor does not pass through the center of mass, which leads to the rotor bearing interference torque, especially in the process of rotor tilt. Interference torque leads to rotor tilt angle jitter and seriously reduces the suspension accuracy of the rotor.

According to the above analysis, the main reason why the rotor bears interference torque is that the force exerted by the rotor does not point to the center of mass, which leads to the existence of force arms between forces and generating interference torque $[19,20]$. The disturbance torque causes the rotor to vibrate in the stable suspension process.

Magnetic suspended control and sensitive gyro (MSCSG) is a new kind of magnetic suspension inertia actuator [21-24]. Integrating the function of attitude angle rate measurement and attitude control, the attitude angle rate measurement and control of spacecraft are realized. In order to improve the attitude measurement accuracy of MSCSG and the accuracy of external output torque, the MSCSG rotor requires high suspension accuracy. The rotor with traditional cylindrical structure cannot meet the requirement of high-precision attitude measurement. Based on the above analysis, the paper proposes a magnetic bearing with pure spherical magnetic pole structure. The characteristic of this structure is that both the stator and rotor of magnetic bearing adopt spherical structure. The electromagnetism force of the rotor points to the spherical center of the rotor, so there is no force arm between the electromagnetism force of the rotor. This structure eliminates the existence of interference torque. In addition, compared with cylindrical rotor, spherical rotor has difficulty in machining, which will lead to imbalance of rotor quality. Because the suspension of the rotor is controlled by the active magnetic bearing, the harmonic interference caused by the mass imbalance during the rotor rotation can be eliminated by the active vibration control of the magnetic bearing $[25,26]$.

Spherical magnetic bearings have been successfully used in MSCSG. 3-DOF translational motion of MSCSG uses full active spherical magnetic bearing. The stator magnetic pole and rotor magnetic pole of magnetic bearing are both spherical structures and the center of the stator magnetic pole and the rotor magnetic pole is coincident. The advantage of this structure is that it can eliminate the disturbance torque of rotor tilt in the process of machining and assembling. Therefore, this structure can effectively improve the rotor suspension precision and further enhance the working characteristics of the MSCSG.

\section{Analysis of Electromagnetic Torque Model of Spherical Magnetic Bearing}

The structure of MSCSG is shown in Figure 1. It mainly consists of radial magnetic bearing, axial magnetic bearing, Lorentz force magnetic bearing (LFMB), double spherical magnetic suspension rotor (the radial magnetic bearing rotor magnetic pole is spherical and axial magnetic bearing rotor magnetic pole is spherical), upper gyro room, middle gyro room, lower gyro room, motor, radial sensor, and axial sensor.

In order to make the whole structure have a better magnetic property, the axial magnetic bearing stator and radial magnetic bearing stator are made of 1550 material with excellent magnetic property, and the magnetic suspension rotor is made of $\mathrm{FeCr}_{15}$ with high strength and excellent magnetic property. The radial magnetic bearing and axial magnetic bearing which control rotor steady suspension of 3-DOF all adopt spherical magnetic pole structure.

In the case of ignoring magnetic flux leakage, the structure diagram and equivalent magnetic circuit diagram of axial magnetic bearing are shown in Figure 2. The structure diagram and equivalent magnetic circuit diagram of radial magnetic bearing are shown in Figure 3, where $I$ is the coil current, $N$ is the coil turns, $N I$ is the magnetomotive force generated by the coil of axial/radial magnetic bearing, $R_{n}$ is the equivalent resistance of the inner ring of axial magnetic bearing, $R_{w}$ is the equivalent resistance of the outer ring of axial magnetic bearing, and $R_{s}$ is stator core reluctance of radial magnetic bearing. $R$ is the air gap resistance between the axial/radial magnetic bearing stator and the rotor.

According to the magnetic path analysis of radial magnetic bearings and axial magnetic bearings, taking the radial magnetic bearing in the $y$ axis as an example, the schematic diagram of the interference torque generated by the magnetic bearing is shown in Figure 4.

As shown in Figure 4, the black outline represents a spherical structural magnetic bearing, and the red outline represents a conventional cylindrical structural magnetic bearing. The red arrow represents the suction of the cylindrical pole stator to the rotor, and the suction force is represented by $F_{y 1}, F_{y 2}, F_{y 3}$, and $F_{y 4}$. It can be seen from the above figure that when the magnetic bearing adopts a spherical structure, the force of the rotor always points to the spherical phase, which is directed to the center of the rotor, and the rotor is free from disturbance torque. By controlling the magnetic bearing coil current, the resultant force of the rotor at the equilibrium position is zero so that it is stably suspended in the equilibrium position.

When the magnetic bearing magnetic pole adopts the cylindrical structure, the force exerted on the rotor points to the normal phase of the rotor surface. At this time, the suction force generated by the stator magnetic pole of the $Y$ direction on the rotor is not on the same straight line, and a 


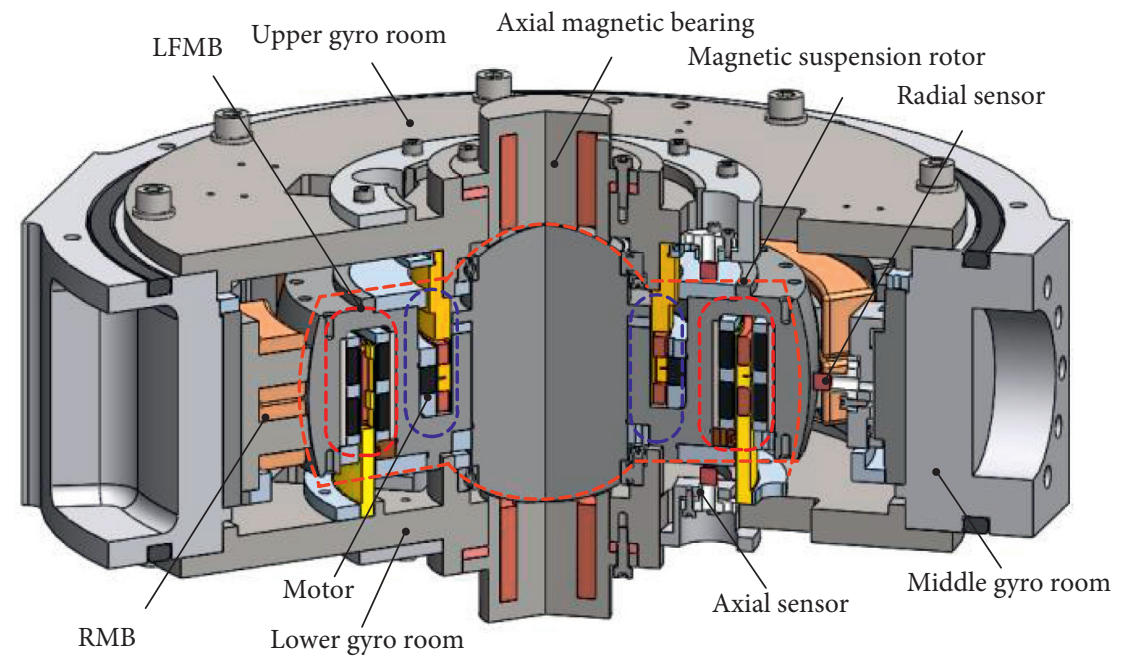

FIGURE 1: Cross section view of MSCSG entirety.

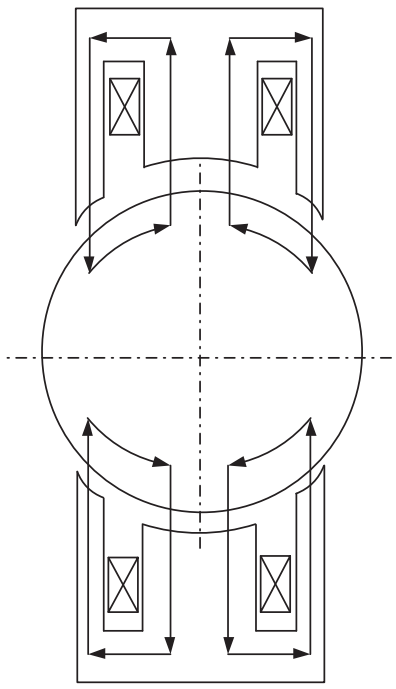

(a)

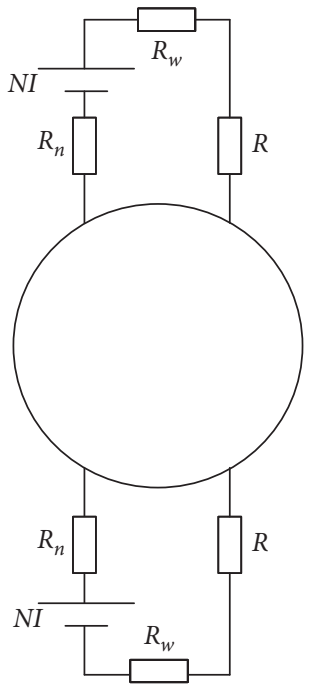

(b)

FIgURE 2: Magnetic circuit analysis of spherical axial magnetic bearing: (a) magnetic circuit diagram of axial magnetic bearing and (b) equivalent magnetic circuit diagram of axial magnetic bearing.

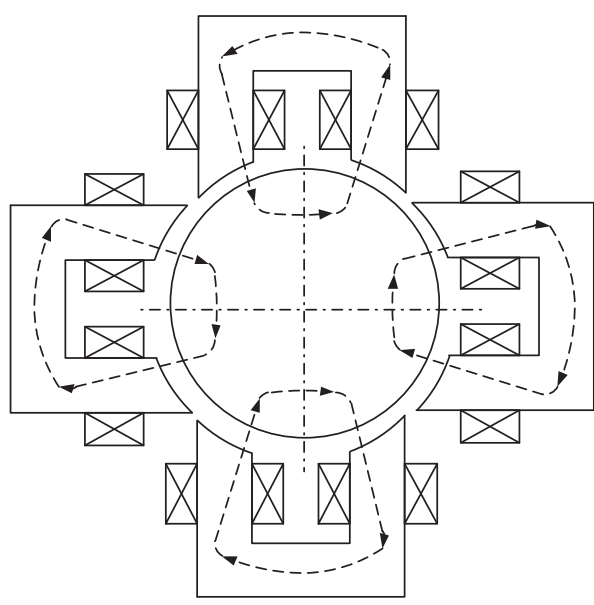

(a)

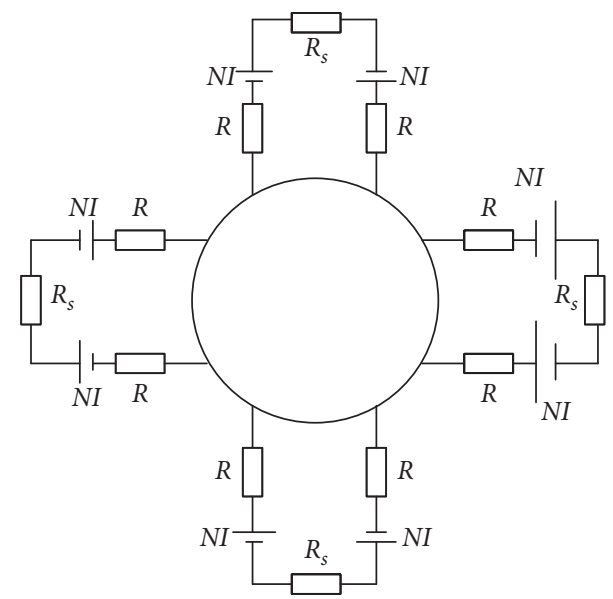

(b)

FIGURE 3: Magnetic circuit analysis of spherical radial magnetic bearing: (a) magnetic circuit of radial magnetic bearing and (b) equivalent magnetic circuit of radial magnetic bearing. 


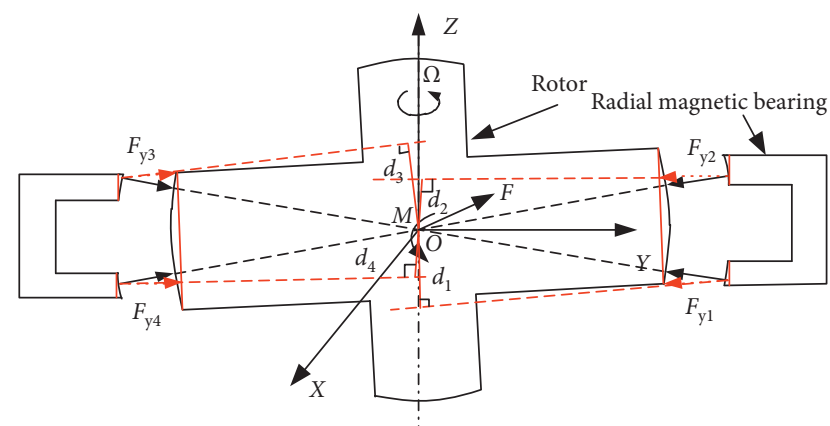

FIgURE 4: Schematic of magnetic bearing interference torque.

force arm is generated between the respective forces. Therefore, the disturbance torque $M(o)$ is generated. The disturbance torque can be expressed as follows:

$$
M_{(o)}=F_{y 1} d_{1}+F_{y 3} d_{3}-F_{y 2} d_{2}-F_{y 4} d_{4},
$$

where $d_{1}, d_{2}, d_{3}$, and $d_{4}$ are force arms in which the stator generates suction force with respect to the rotor core, and their value changes with the tilt angle of the rotor.

In the above formula, $F_{y 1}, F_{y 2}, F_{y 3}$, and $F_{y 4}$ can be expressed as follows:

$$
\left[\begin{array}{c}
F_{y 1} \\
F_{y 2} \\
F_{y 3} \\
F_{y 4}
\end{array}\right]=\left[\begin{array}{l}
k_{i} i_{1}-k_{s} x_{1} \\
k_{i} i_{2}-k_{s} x_{2} \\
k_{i} i_{3}-k_{s} x_{3} \\
k_{i} i_{4}-k_{s} x_{4}
\end{array}\right],
$$

where $K_{i}$ represents the current stiffness of the radial magnetic bearing, $K_{s}$ represents the displacement stiffness of radial magnetic bearing, $i$ is the control current applied by the radial magnetic bearing, and $x$ represents the magnetic gap between the stator and the rotor.

According to the synthesis theorem of the plane force system, the plane force system of the rotor can be simplified into a force couple $M$ and a resultant force $F$.

The force couple $M$ affects the tilt accuracy of the rotor, that is, the tilt angle of the rotor fluctuates. The resultant force $F$ affects the positional accuracy of the rotor.

In this paper, ANSYS finite element analysis software is used to analyze magnetic field. The three-dimensional finite element model and magnetic field distribution of the axial magnetic bearing are shown in Figure 5, and the radial magnetic bearing is shown in Figure 6.

It can be seen from Figure 7 that when the rotor adopts a spherical structure, by comparing the magnetic field on the surface of the rotor which is not tilted at the balance position and which tilted 0.3 degree of rotor, the magnetic field on the surface of the rotor is basically unchanged, so the force on the rotor remains unchanged. Therefore, the analysis of the magnetic field distribution on the spherical rotor surface shows that the spherical magnetic bearing can eliminate the interference torque when the rotor tilts.

Through finite element simulation analysis, when the rotor is tilted, the interference torque generated by the spherical magnetic bearing and the cylindrical magnetic bearing at the equilibrium position and the inclination of the rotor are shown in Figure 8.

According to the analysis of the disturbance torque of magnetic suspension rotor, when the rotor is tilted between $0 \sim 0.3^{\circ}$, the disturbance torque of cylindrical magnetic bearing is positively correlated with the increase of rotor tilt angle. When the rotor tilt angle is $0.3^{\circ}$, interference torque is about $14 \mathrm{Nm}$ produced by cylindrical magnetic bearings.

However, the disturbance torque of spherical magnetic bearing does not change with the change of tilt angle of magnetic suspension rotor, and the disturbance torque basically remains $0 \mathrm{Nm}$. Therefore, by comparison, it is found that the disturbance torque of cylindrical magnetic bearing cannot be ignored, while the interference torque of spherical magnetic bearing can be ignored. So, the spherical magnetic bearing can eliminate the disturbance torque and improve the overall performance of the magnetic inertia mechanism.

\section{Experimental Results and Discussion}

Physical diagram of the radial magnetic bearing stator and the axial magnetic bearing stator is shown in Figure 9. It can be observed that the stator magnetic pole of radial magnetic bearing is a spherical structure, and a closed magnetic circuit is formed by a double-layer stator structure. Axial magnetic bearing stator magnetic pole adopts spherical structure. The spherical magnetic pole rotor and size drawing are shown in Figure 10. The magnetic suspension rotor adopts double-sphere structure. The double-sphere is that the rotor magnetic pole of radial magnetic bearing and axial magnetic bearing adopt spherical structure. In addition, the rotor magnetic pole surface of radial magnetic bearing and the rotor magnetic pole surface of axial magnetic bearing have the same center of sphere. The main parameters of magnetic suspension rotor are shown in Table 1.

The prototype experiment system of MSCSG with fully active spherical magnetic bearing structure is shown in Figure 11. It is seen clearly that the experimental system mainly includes principle prototype of MSCSG, magnetic bearing control circuit, motor control circuit, the oscilloscope, power supply, and debugging computer. In this system, MSCSG is the experimental object. The main function of the circuit control board controls the suspension and rotation of the rotor. The function of power provides energy to the system. The oscilloscope mainly collects experimental data, and the main function of debug computer is to input control instructions and control the whole system. The MSCSG control system adopts classic PID control, and its control flow is shown in Figure 12.

The mounting position of the sensor used for detecting the displacement signal and angle displacement signal of the spherical magnetic suspension rotor is shown in Figure 13. It includes 8 axial displacement sensors and 4 


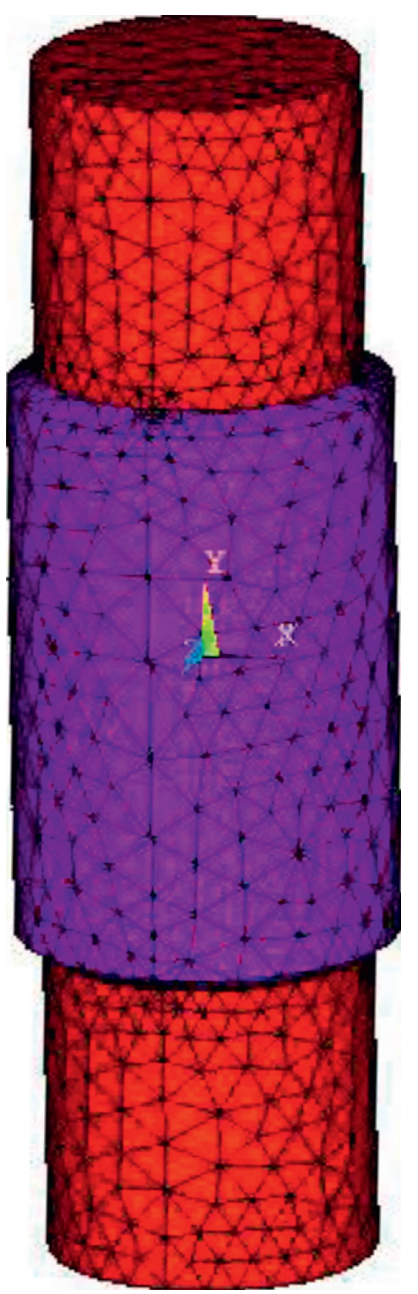

(a)

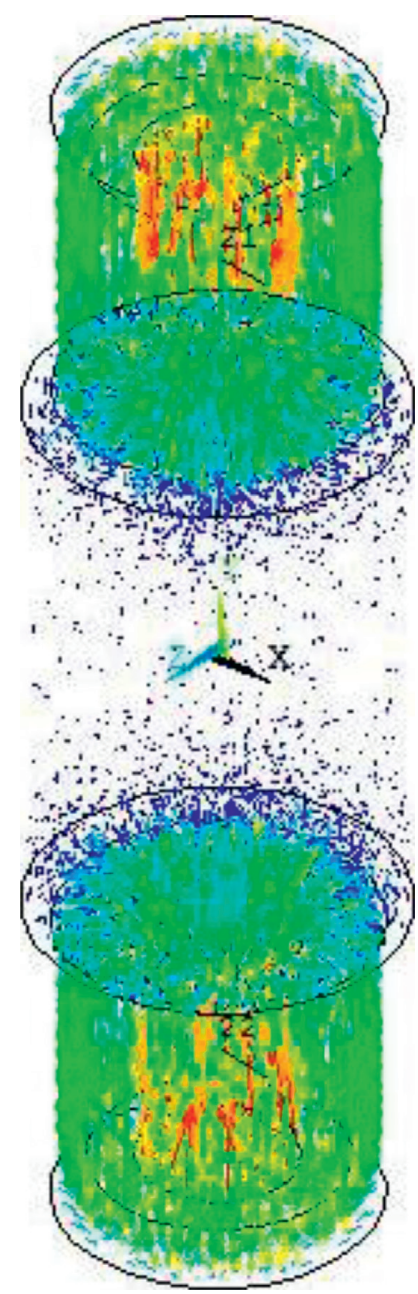

(b)

Figure 5: Three-dimensional finite element analysis of the spherical axial magnetic bearing: (a) the three-dimensional finite element model of spherical active axial magnetic bearing and (b) magnetic field distribution of the axial magnetic bearing.

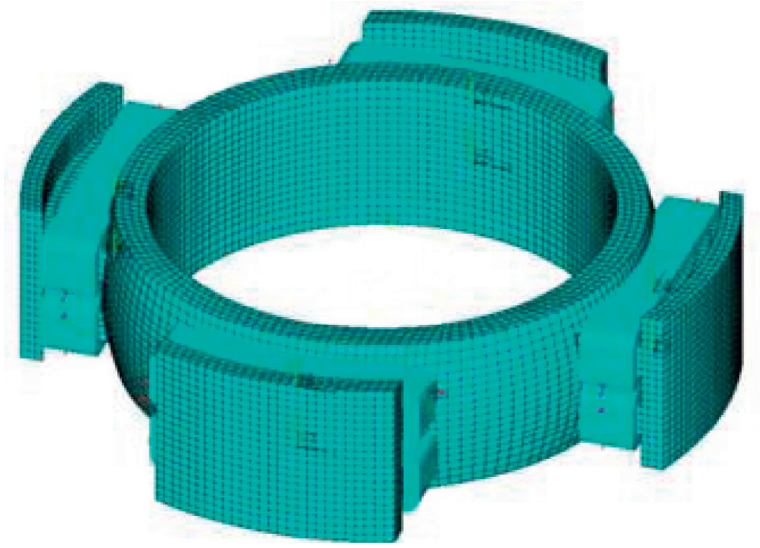

(a)

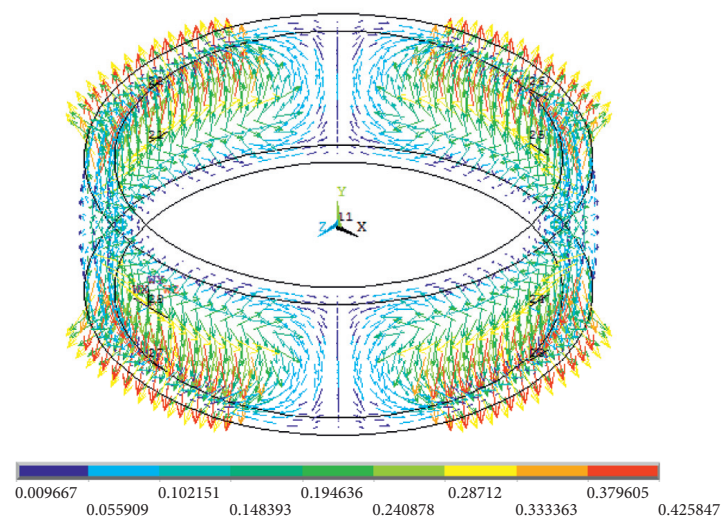

(b)

FIGURE 6: Three-dimensional finite element analysis of the spherical radial magnetic bearing: (a) the three-dimensional finite element model of spherical radial magnetic bearing and (b) magnetic field distribution of the radial magnetic bearing. 


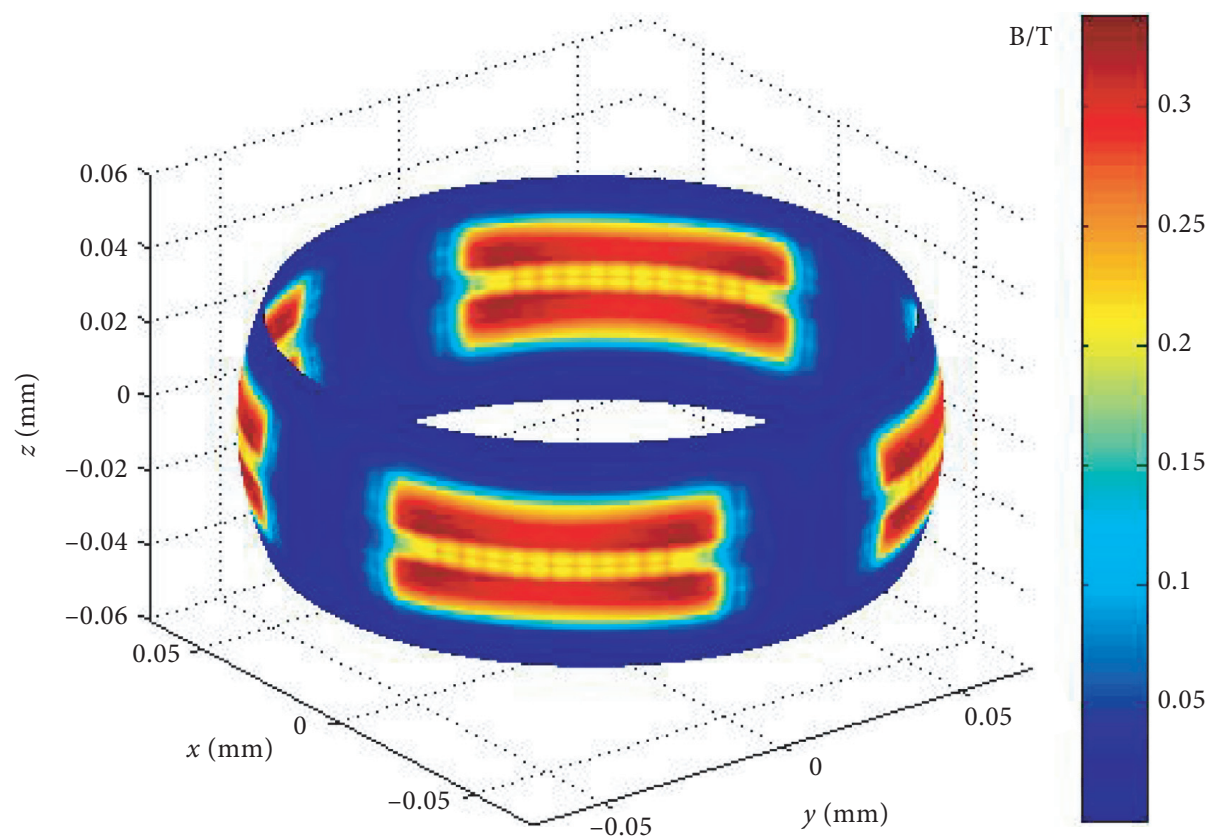

(a)

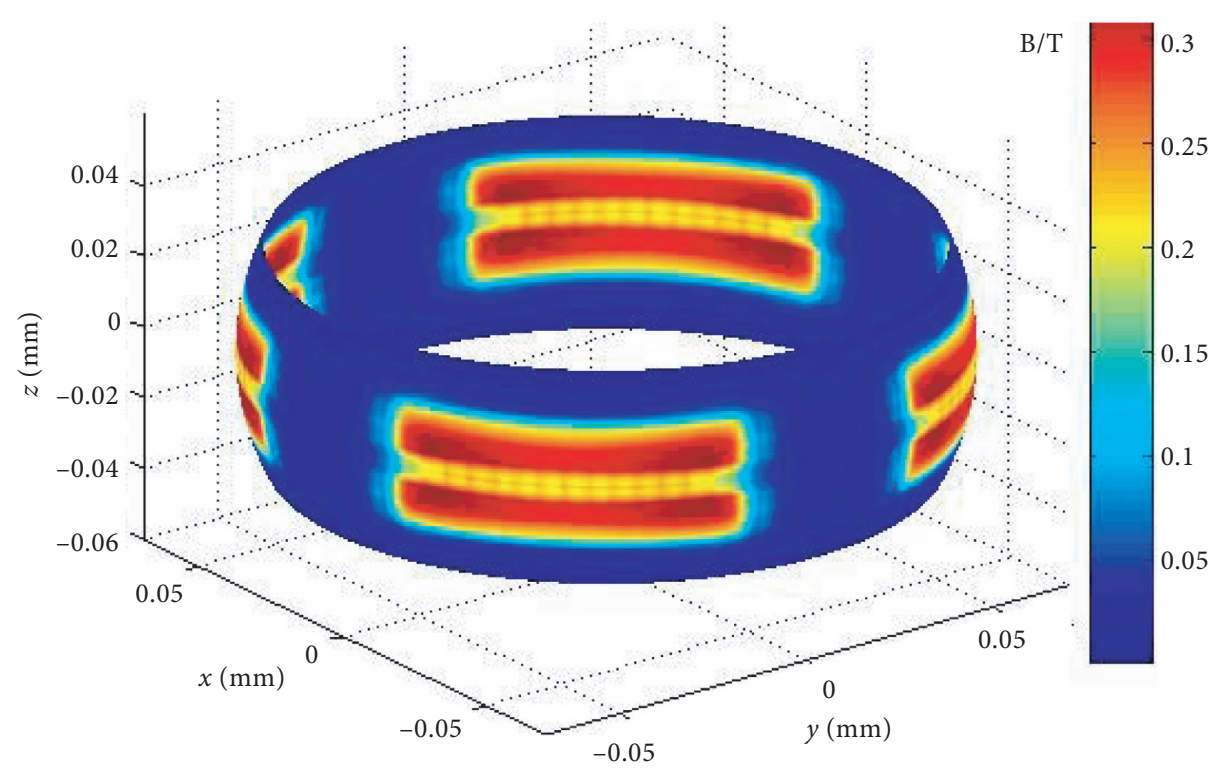

(b)

FIgURe 7: The magnetic field intensity distribution diagram of the radial spherical rotor surface: (a) the spherical magnetic field intensity distribution diagram when the rotor is not tilted and (b) the spherical magnetic field distribution diagram when the rotor is tilted by $0.3^{\circ}$.

radial displacement sensors. 4 axial displacement sensors are distributed at the upper end of the rotor, and 4 axial displacement sensors are distributed at the lower end of the rotor. Radial sensors are evenly distributed at $90^{\circ}$ around the circumference of the rotor. The axial displacement sensor detects the axial displacement signal of the rotor by detecting the displacement detection plate. The radial displacement sensor detects the radial sphere of the rotor. The axial and radial displacement signals of the rotor can be expressed as follows:

$$
\left\{\begin{array}{l}
z=\frac{1}{2}\left(\frac{1}{4}\left(A_{1}+A_{2}+A_{3}+A_{4}\right)-\frac{1}{4}\left(A_{5}+A_{6}+A_{7}+A_{8}\right)\right) \\
x=\frac{1}{2} \cos \left(R_{1}-R_{3}\right) \\
y=\frac{1}{2} \cos \left(R_{2}-R_{4}\right) .
\end{array}\right.
$$




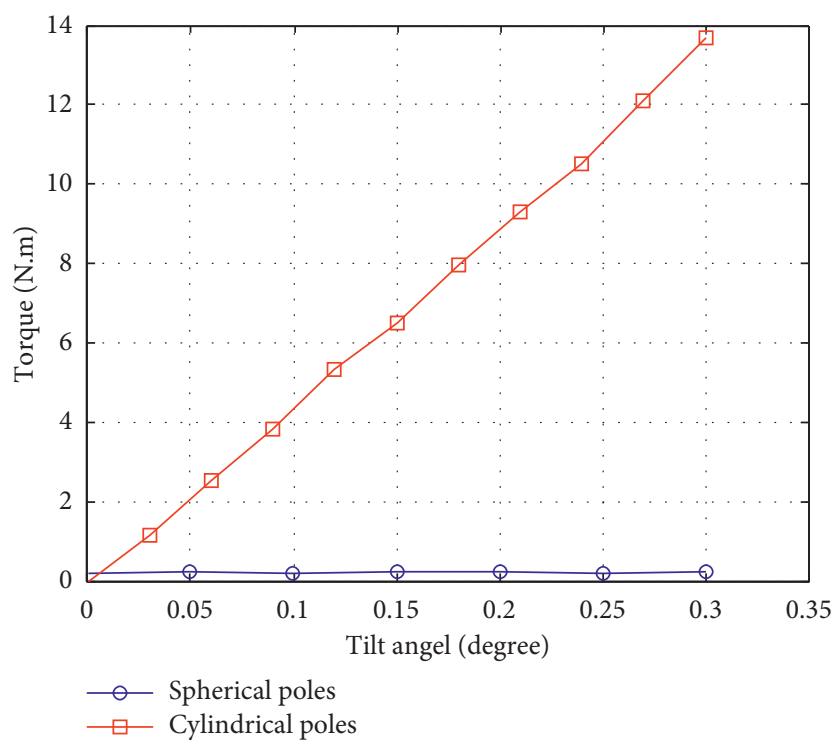

FIGURE 8: Relationship between the tilt angle of magnetic suspension rotor and the disturbance torque. The red line represents the disturbance torque of the rotor with cylindrical magnetic bearing, while the blue line represents the disturbance torque of the rotor with spherical magnetic bearing.

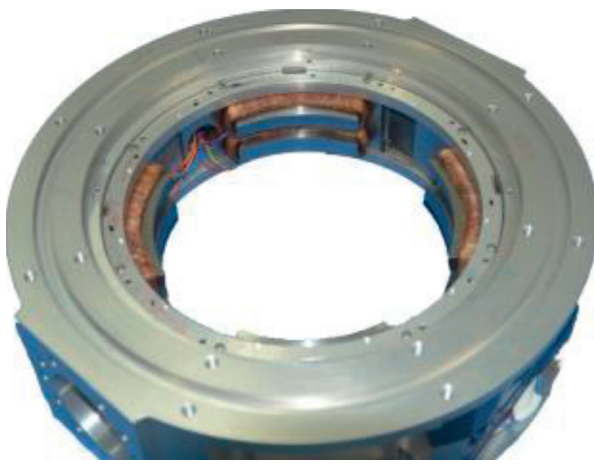

(a)

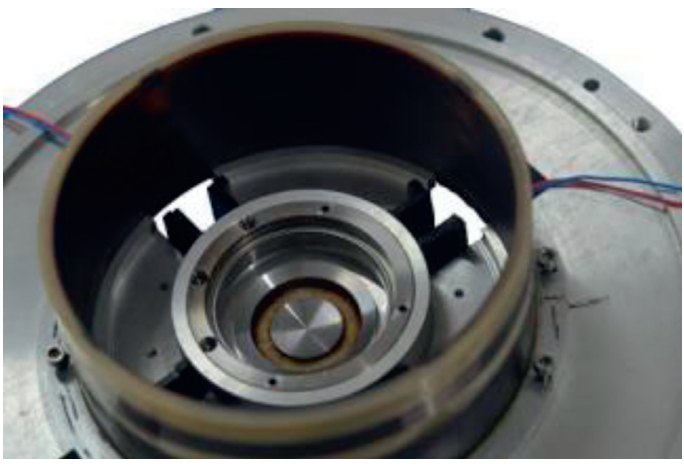

(b)

FIGURE 9: Stator of spherical magnetic bearing: (a) spherical pole of radial magnetic bearing stator and (b) spherical pole of axial magnetic bearing stator.

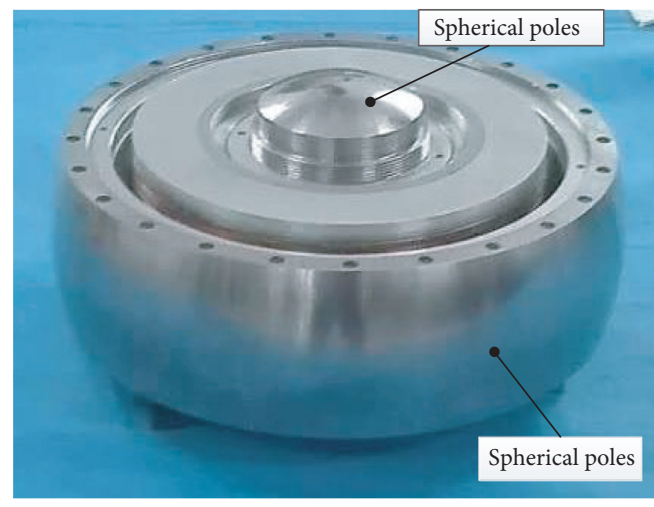

(a)

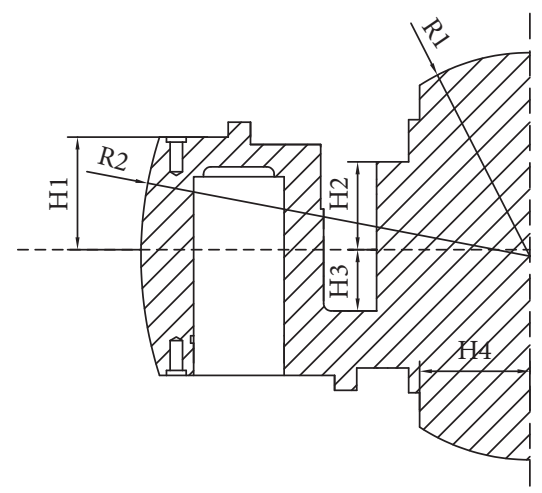

(b)

FIGURE 10: Magnetic suspension rotor: (a) physical picture of magnetic suspension rotor and (b) size picture of magnetic suspension rotor. 
TABLE 1: The main parameters of magnetic suspension rotor.

\begin{tabular}{lcll}
\hline$R 1(\mathrm{~mm})$ & 41 & $H 2(\mathrm{~mm})$ & 20.5 \\
$R 2(\mathrm{~mm})$ & 78.65 & $H 3(\mathrm{~mm})$ & 13.5 \\
$H 1(\mathrm{~mm})$ & 24 & $H 4(\mathrm{~mm})$ & 22.3 \\
\hline
\end{tabular}

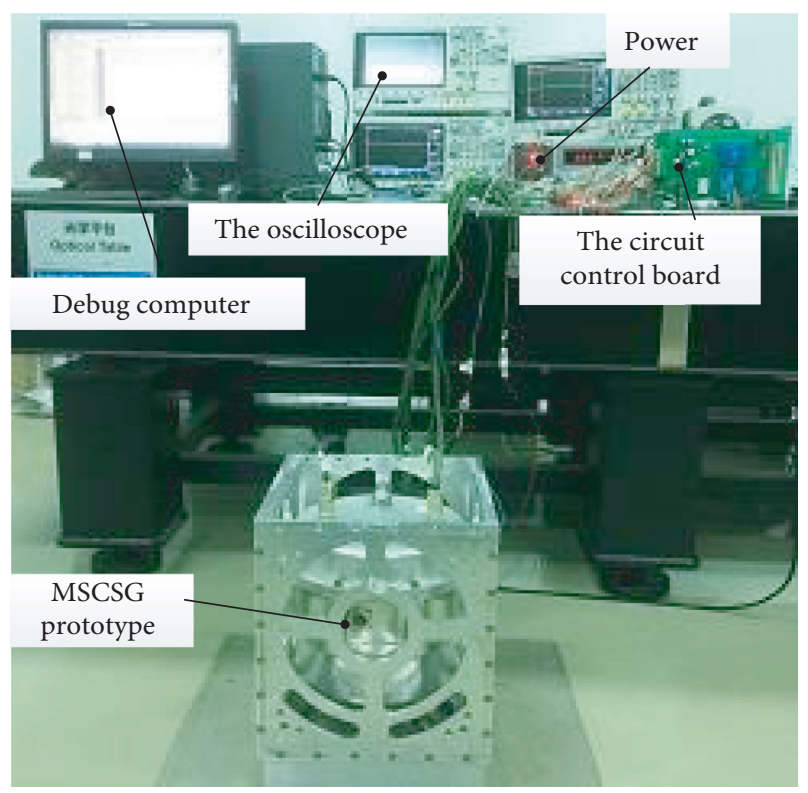

Figure 11: MSCSG experimental system.

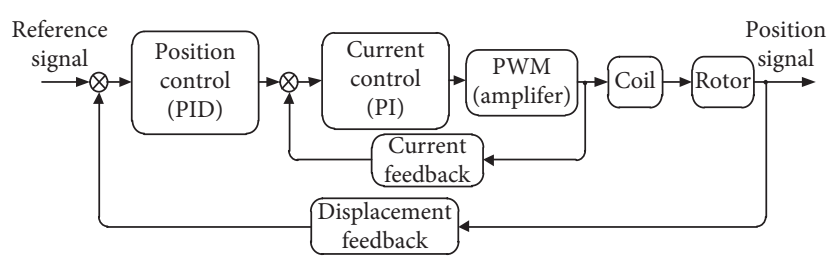

Figure 12: MSCSG Control system block diagram.

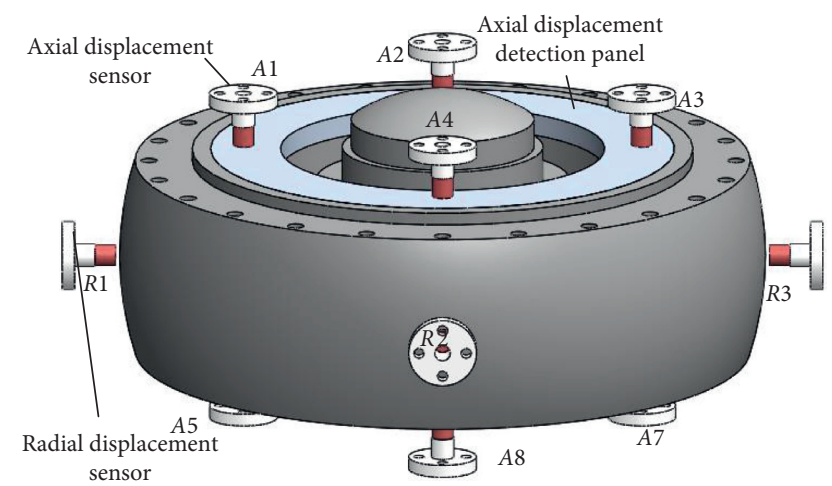

FIgURE 13: Displacement sensor array.

The rotor tilted around the $X$-axis is defined as angle of $\alpha$, and the rotor tilted around the $Y$ axis is defined as an angle of $\beta$. The tilt signal of the rotor can be calculated by the axial displacement sensor as follows:

$$
\left\{\begin{array}{l}
\tan \alpha=\frac{1 / 2\left(A_{1}-A_{5}\right)-1 / 2\left(A_{3}-A_{7}\right)}{L_{s}} \\
\tan \beta=\frac{1 / 2\left(A_{2}-A_{6}\right)-1 / 2\left(A_{4}-A_{8}\right)}{L_{s}} .
\end{array}\right.
$$

The axial displacement sensor is distributed in a circle. In the above equation, $L s$ is the circumference diameter. $L s=100 \mathrm{~mm}$, and $A_{i}(i=1,2, \ldots, 8)$ represents the axial displacement of the rotor. $-0.2 \mathrm{~mm}<A_{i}<0.2 \mathrm{~mm}$. Because $L_{s}$ is much larger than the signal detected by the axial displacement sensor,

$$
\left\{\begin{array}{l}
\alpha \approx \frac{1 / 2\left(A_{1}-A_{5}\right)-1 / 2\left(A_{3}-A_{7}\right)}{L_{s}}, \\
\beta \approx \frac{1 / 2\left(A_{2}-A_{6}\right)-1 / 2\left(A_{4}-A_{8}\right)}{L_{s}} .
\end{array}\right.
$$

In order to better describe the influence of rotor disturbance torque, the concepts of displacement runout and angle displacement runout are given in this paper. The displacement runout is the difference between the displacement signal of the rotor and the signal of the rotor at the balance position during the rotor tilting. Angle displacement runout is the difference between the angle displacement signal of the rotor and the angle displacement of the rotor at the equilibrium position during the rotor tilting process.

The displacement runout $K_{d}$ and angle displacement runout $K_{\alpha}$ are defined as follows:

$$
\left\{\begin{array}{l}
K_{d}=R_{r}-R_{i} \\
K_{\alpha}=A_{r}-A_{i}
\end{array}\right.
$$

where $R_{r}$ represents the actual displacement of the rotor, $R_{i}$ represents the theoretical displacement of the rotor, $A_{r}$ represents the actual angle displacement of the rotor, and $A_{i}$ represents the theoretical angle displacement of the rotor.

The interference torque of the magnetic suspension rotor cannot be directly measured through the experiment, but the interference torque of the magnetic suspension rotor will cause the rotor to shake in the tilted state, so the interference torque of the rotor can be obtained by detecting the displacement and angle displacement signals of the magnetic suspension rotor. The specific experimental settings are as follows: the rotor displacement signal and angle displacement signal are collected by an oscilloscope, and the acquisition frequency of an oscilloscope is $2 \mathrm{GHz}$. When the rotor is in the equilibrium position, the rotor has no interfering torque, and the noise signal collected by the oscilloscope is mainly caused by electromagnetic interference. Then, gradually increase the rotor tilt angle; at this time, the oscilloscope collected rotor displacement and angle displacement signal's runout amount mainly caused by electromagnetic interference, noise interference, and interference torque. By comparing the runout of the displacement and angle displacement signals in the rotor tilt process with the runout of the displacement and angle 


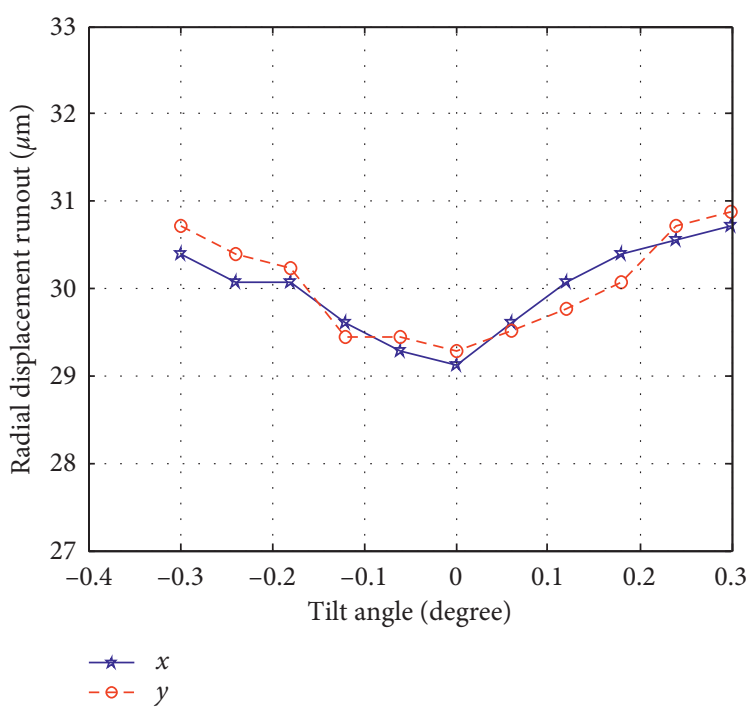

(a)

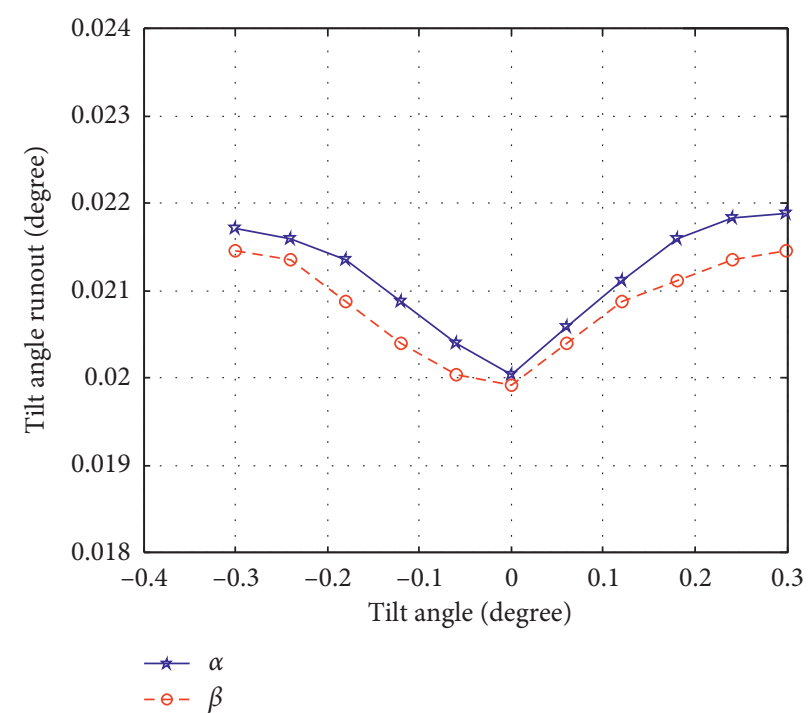

(b)

FIgURE 14: Experimental results of rotor interference torque.

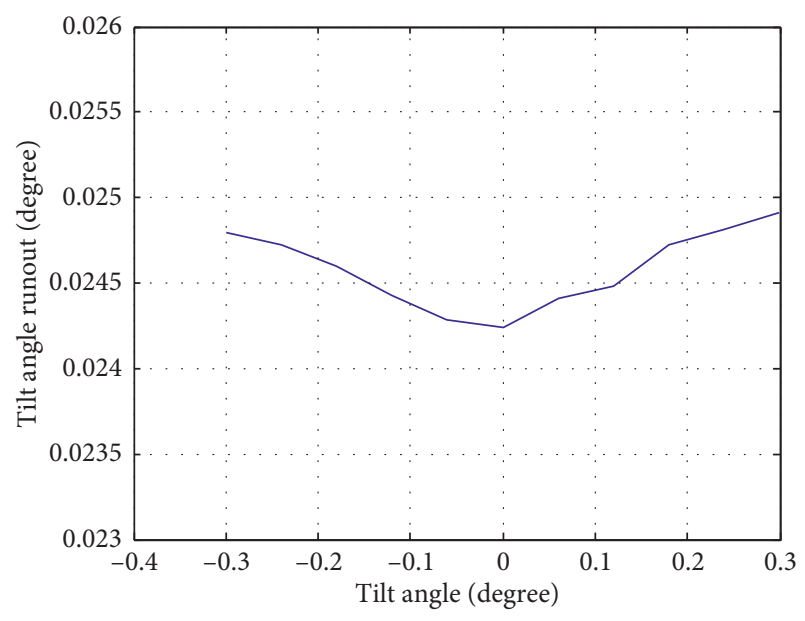

FIGURE 15: Experimental results of rotor interference torque $(83.33 \mathrm{~Hz})$.

displacement signals in the balance position, the interference torque to the rotor is observed.

The oscilloscope collects the sensor's signal and obtains the rotor's displacement signal and tilt angle signal through the formulas (2) and (3). The displacement runout and angle displacement runout of the rotor are obtained by formula (6).

The experimental results are shown in Figure 14. Figure 14(a) shows the relation between the rotor radial displacement runout value and the rotor tilt angle. Figure 14(b) shows the relationship between the rotor tilt angle runout value and the tilt angle.

As shown in Figure 14, the experimental condition is that the rotor tilts gradually from the equilibrium position about the $X$ axis (increasing the angle $\alpha$ ). The rotor radial displacement and the tilt angle runout amount were observed during the tilting of the rotor.
According to Figure 14(a), when the rotor is in the equilibrium position (tilt angle $=0^{\circ}$ ), the radial displacement runout value of the rotor with radial two degrees of freedom is $x=29.12 \mu \mathrm{m}$ and $y=29.28 \mu \mathrm{m}$. In the process of rotor tilt (tilt angle increases gradually), the radial displacement runout value is basically consistent with balance position of the rotor with radial two degrees of freedom. The maximum runout of radial two degrees of freedom is $x=30.72 \mu \mathrm{m}$ and $y=30.88 \mu \mathrm{m}$. Compared with the equilibrium position (tilt angle $=0$ ), the maximum deviation from the equilibrium position in the $X$ direction is $1.6 \mu \mathrm{m}$ and the maximum deviation from the equilibrium position in the $Y$ direction is $1.6 \mu \mathrm{m}$. The error caused by the interference torque is $5.49 \%$ in the $X$ direction and $5.46 \%$ in the $Y$ direction.

Figure 14(b) shows that when the rotor is in the equilibrium position (tilt angle $=0^{\circ}$ ), the two degrees of freedom rotor tilting runout value is $\alpha=0.0199^{\circ}$ and $\beta=0.0200^{\circ}$. In the process of rotor tilting, the runout value of rotor tilt angle is basically consistent with the balance position (tilt angle $=0^{\circ}$ ). The maximum runout value of $\alpha$ is $0.0215^{\circ}$. The maximum runout value of $\beta$ is $0.0219^{\circ}$. Compared to the equilibrium position, the maximum error of the tilt angle $\alpha$ is $0.0016^{\circ}$. deg and the maximum error of the tilt angle $\beta$ is $0.0019^{\circ} \mathrm{deg}$. The error of the interference torque to the angle of $\alpha$ is $8.04 \%$, and the error to the angle of $\beta$ is $9.5 \%$.

By comparing the runout value, it can be seen that the suspension result of the rotor in the tilt process is consistent with the state when the rotor does not tilt (tilt angle $=0^{\circ}$ ), so the spherical magnetic bearing will not generate interference torque to the rotor.

In order to show that the rotor can also eliminate the interference torque in the dynamic state, when the rotor is at the rated speed $(83.33 \mathrm{~Hz})$, the rotor tilt angle is controlled and the runout value of the tilt angle is observed. The experimental results are shown in Figure 15. 
The experimental conditions in Figure 15 are as follows: under the condition of rated speed $(83.33 \mathrm{~Hz})$, the tilt angle of $\alpha$ is gradually increased. Observe tilt angle runout. The experimental result shows that when the rotor is in the equilibrium position (tilt angle $=0^{\circ}$ ), the rotor tilt angle of $\alpha$ runout value is $0.0242^{\circ}$. When changing the tilt angle of the rotor position, rotor maximum tilt angle runout value $\alpha$ is $0.0249^{\circ}$. Its biggest deviation from the equilibrium position is $0.0007^{\circ}$. The error of interference torque to $\alpha$ is $2.89 \%$. The experimental results show that the spherical magnetic bearing will not cause interference torque to the rotor during dynamic operation.

\section{Conclusion}

This paper presents a spherical radial and axial magnetic bearing. The biggest advantage of this structure is that it can eliminate the interference torque. Firstly, this paper analyzes the reasons for the interference torque produced by spherical magnetic bearing and traditional cylindrical magnetic bearing. Through analysis, it can be seen that spherical magnetic bearing can eliminate the interference torque generated in the process of rotor tilting. Through finite element simulation, it is found that the interference torque of cylindrical magnetic bearing increases with the increase of rotor tilting, and the interference torque of spherical magnetic bearing basically remains at $0 \mathrm{Nm}$ with the rotor tilt. Finally, the interference torque produced by spherical magnetic bearing is verified by experiment. In the process of rotor tilting, its displacement signal and tilt angle signal are consistent with the balance state (tilt angle $=0^{\circ}$ ). It is found from experiments that the spherical magnetic bearing can eliminate the interference torque in the process of rotor tilt.

The spherical magnetic bearing proposed in this paper has important reference significance for improving the performance of magnetic suspension inertia mechanism.

\section{Data Availability}

The data used to support the findings of this study were supplied by Yin Zengyuan under license and so cannot be made freely available. Requests for access to these data should be made to Yin Zengyuan.

\section{Conflicts of Interest}

The authors declare that they have no conflicts of interest.

\section{Acknowledgments}

This study was supported by the National Natural Science Foundation of China under grant nos. 51605489 and 51475472.

\section{References}

[1] H. Wang, K. Liu, P. Ao et al., "Magnetic field and specific axial load capacity of hybrid magnetic bearing," IEEE Transactions on Magnetics, vol. 49, no. 8, pp. 4911-4917, 2013.

[2] J. Sun, Y. C. E. Wang, and Y. Le, "Research on a novel high stiffness axial passive magnetic bearing for DGMSCMG,"
Journal of Magnetism and Magnetic Materials, vol. 412, pp. 147-155, 2016.

[3] H. Eryong, "Analytical calculation of magnetic field and force for hybrid magnetic bearing," Journal of Mechanical Engineering, vol. 48, no. 6, p. 193, 2012.

[4] S. Zheng, Y. B. Han, and J. Zhou, "Optimization of damping compensation for a flexible rotor system with active magnetic bearing considering gyroscopic effect," IEEE/ASME Transactions on Mechatronics, vol. 20, no. 3, pp. 1130-1137, 2015.

[5] B. Han, Z. S. Zheng, and Y. Le, "Design, modeling, fabrication, and test of a large-scale single-gimbal magnetically suspended control moment gyro," IEEE Transactions on Industrial Electronics, vol. 62, no. 12, pp. 7424-7435, 2015.

[6] J. Sun, Z. Ju, W. Han et al., "A novel integrated 4-DOF radial hybrid magnetic bearing for MSCMG," Journal of Magnetism \& Magnetic Materials, vol. 421, pp. 86-97, 2017.

[7] B. Han, S. Zheng, W. Xi, and Q. Yuan, "Integral design and analysis of passive magnetic bearing and active radial magnetic bearing for agile satellite application," IEEE Transactions on Magnetics, vol. 48, no. 6, pp. 1959-1966, 2012.

[8] T. Azukizawa, S. Yamamoto, and N. Matsuo, "Feasibility study of a passive magnetic bearing using the ring shaped permanent magnets," IEEE Transactions on Magnetics, vol. 44, no. 11, pp. 4277-4280, 2008.

[9] J.-H. Liu, X.-K. Yang, H.-Q. Cui et al., "Modeling of $180^{\circ}$ magnetization switching and clock sensitivity in a tilted multiferroic nanomagnet," Journal of Magnetism and Magnetic Materials, vol. 474, pp. 161-166, 2019.

[10] P. Samanta and H. Hirani, "Magnetic bearing configurations: theoretical and experimental studies," IEEE Transactions on Magnetics, vol. 44, no. 2, pp. 292-300, 2008.

[11] T. Ohji, S. Ichiyama, K. Amei et al., "A new conveyor system based on a passive magnetic levitation unit having repulsivetype magnetic bearings," Journal of Magnetism and Magnetic Materials, vol. 272-276, pp. e1731-e1733, 2004.

[12] J. M. D. Coey, "Permanent magnet applications," Journal of Magnetism and Magnetic Materials, vol. 248, no. 3, pp. 441456, 2002.

[13] J. Tang, J. J. Sun, and G. Shuzhi Sam, "Low eddy loss axial hybrid magnetic bearing with gimballing control ability for momentum flywheel," Journal of Magnetism and Magnetic Materials, vol. 329, pp. 153-164, 2013.

[14] S. Wen and S. Jiang, "Optimum design of hybrid composite multi-ring flywheel rotor based on displacement method," Composites Science and Technology, vol. 72, no. 9, pp. 982-988, 2012.

[15] E. Hou and K. Liu, "A novel structure for low-loss radial hybrid magnetic bearing," IEEE Transactions on Magnetics, vol. 47, no. 12, pp. 4725-4733, 2011.

[16] J. Fang, C. Wang, and T. Wen, "Design and optimization of a radial hybrid magnetic bearing with separate Poles for magnetically suspended inertially stabilized platform," IEEE Transactions on Magnetics, vol. 50, no. 5, pp. 1-11, 2014.

[17] S. Xu and J. Fang, "A novel conical active magnetic bearing with claw structure," IEEE Transactions on Magnetics, vol. 50, no. 5, pp. 1-8, 2014.

[18] C. Wang, Design Method and Experimental Research on High Specific Load Capacity and Low Loss Magnetic Bearing in Inertia Actuator, Beijing University of Aeronautics and Astronautics, Beijing, China, 2018.

[19] J. Fang and Y. Ren, "High-precision control for a singlegimbal magnetically suspended control moment gyro based on inverse system method," IEEE Transactions on Industrial Electronics, vol. 58, no. 9, pp. 4331-4342, 2011. 
[20] L. Zhang, J. Du, and J. Feng, "Control for the magnetically suspended flat rotor tilting by axial forces in a small-scale control moment gyro," IEEE Transactions on Industrial Electronics, vol. 65, no. 3, pp. 2449-2457, 2018.

[21] F. Jiancheng, L. S. Jinji, and T. Jiqiang, "A novel 3-DOF axial hybrid magnetic bearing," IEEE Transactions on Magnetics, vol. 46, no. 12, pp. 4034-4045, 2010.

[22] X. Chang-Feng, C. Yuan-Wen, R. Yuan et al., "Stability analysis method with extended double-frequency bode diagram for rotor of MSCSG," Journal of Astronautics, vol. 39, no. 2, pp. 168-176, 2018.

[23] Y. Ren, X. Chen, Y. Cai, H. Zhang, C. Xin, and Q. Liu, "Attitude-rate measurement and control integration using magnetically suspended control and sensitive gyroscopes," IEEE Transactions on Industrial Electronics, vol. 65, no. 6, pp. 4921-4932, Jun. 2018.

[24] X. Chen, Y. Cai, C. Yang, and X.-D. Peng, "Spacecraft angular rates and angular acceleration estimation using single-gimbal magnetically suspended control moment gyros," IEEE Transactions on Industrial Electronics, vol. 66, no. 1, pp. 440-450, 2019.

[25] S. Zheng and R. Feng, "Feedforward compensation control of rotor imbalance for high-speed magnetically suspended centrifugal compressors using a novel adaptive notch filter," Journal of Sound and Vibration, vol. 366, pp. 1-14, 2016.

[26] Y. Zebin, D. Dawei, S. Xiaodong et al., "Rotor vibration feedforward compensation control in bearingless induction motor based on coordinate transformation," Journal of Central South University (Science and Technology), vol. 47, no. 5, pp. 1143-1149, 2016. 